\title{
Noncommutative Quantum Cosmology
}

\author{
Luis O. Pimentel† and César Mora $\ddagger$ \\ $\dagger$ Departamento de Física, \\ Universidad Autónoma Metropolitana, \\ Apartado Postal 55-534,CP 09340 México D.F., MEXICO. \\ $\ddagger$ Departamento de Matemáticas, \\ UPIBI-Instituto Politécnico Nacional, \\ Av. Acueducto s/n Col. Barrio La Laguna Ticomán, \\ CP 07340 México DF, MEXICO.
}

(Dated: June 22, 2018)

\begin{abstract}
We consider noncommutative quantum cosmology in the case of the low-energy string effective theory. Exacts solutions are found and compared with the commutative case.The Noncommutative quantum cosmology is considered in the case of the low-energy string effective theory. Exacts solutions are found and compared with the commutative case.
\end{abstract}

PACS numbers: 04.60.Kz, 11.10.Lm, 11.25.Sq, 98.80.Hw

\section{INTRODUCTION}

There is a renewed interest on non commutative theories in physics, one of the reasons for that is that noncommutativity between coordinates appears in string theory: in the toroidal compactification of Matrix Theory [1] and in open string theory with a B-field background [2]. Noncommutative classical [4] and quantum mechanics [6] has been considered. The effects of noncommutativity has been studied also in gravity [5]. In the field of quantum cosmology, noncommutativity of the minisuperspace coordinates was introduce by Compean et al. 3]. They studied the Kantowski-Sachs metric and were able to find the exact wave function, with which wave packets were constructed and found new quantum states that "compete" to be the most probable state, in clear contrast with the commutative case. A tunnelling process could be possible among these different states for the Universe. We want to explore the effects of noncommutativity in quantum cosmology when matter fields are included.

\section{THE LOW-ENERGY STRING EFFECTIVE QUANTUM COSMOLOGY}

In this work we want to study noncommutative cosmology with some matter included and not just pure gravity as was the case of [3]. In order to have an exact solution we must consider a geometry with high symmetry, the Robertson-Walker-Friedmann models.For the matter content of our model we will consider the low energy limit of string theory and we will have an scalar field besides the gravitational degrees of freedom. Some other gravitational theories of the scalar-tensor type are under consideration [7]. At low energy, the tree-level, $(3+1)$-dimensional string effective action can be written as [8]

$$
S=-\frac{1}{2 \lambda_{s}} \int d^{4} x \sqrt{-g} e^{-\phi}\left(R+\partial_{\mu} \phi \partial^{\mu} \phi+V\right) .
$$

Here $\phi$ is the dilaton field, $a(t)=\exp [\beta(t) / \sqrt{3}], \lambda_{s}$ is the fundamental string length parameter governing the highderivative expansion of the action and $V$ is a possible dilaton potential. When we consider this theory in the metric of isotropic and homogeneous spacetime, after integrating by parts, and using the convenient time parametrization $d t=e^{-\phi} d \tau$, reduces to (in the gauge $g_{00}=1$ )

$$
S=-\frac{\lambda_{s}}{2} \int d \tau\left(\bar{\phi}^{\prime 2}-\bar{\beta}^{\prime 2}+V e^{-2 \bar{\phi}}\right),
$$

where

$$
\bar{\phi}=\phi-\ln \int\left(d^{3} x / \lambda_{s}^{3}\right)-\sqrt{3} \beta
$$


The Hamiltonian of the system is

$$
H=\frac{1}{2 \lambda_{s}}\left(\Pi_{\beta}^{2}-\Pi_{\phi}^{2}+\lambda_{s}^{2} V e^{2 \bar{\phi}}\right),
$$

where the canonical conjugate momenta are,

$$
\Pi_{\beta}=\lambda_{s} \beta^{\prime}, \quad \Pi_{\phi}=-\lambda_{s} \bar{\phi}^{\prime} .
$$

The corresponding Wheeler-DeWitt equation, in a particular factor ordering is

$$
\frac{1}{2 \lambda_{s}}\left[\frac{\partial^{2}}{\partial \bar{\phi}^{2}}-\frac{\partial^{2}}{\partial \beta^{2}}+\lambda_{s}^{2} V(\bar{\phi}, \beta) e^{-2 \bar{\phi}}\right] \psi(\phi, \beta)=0 .
$$

We shall assume $V=V(\phi)$ in order to separate variables. We consider two simple cases of the potential as toy models that allow us to obtain exact solutions

1) Case: $V=-V_{0} e^{4 \bar{\phi}}$

Therefore the solution of the WDW equation (6) is

$$
\psi_{\nu}(\bar{\phi}, \beta)=C e^{i \nu \beta} Y_{i \nu}\left(\lambda_{s} \sqrt{V_{0}} e^{\bar{\phi}}\right),
$$

where $Y_{i \nu}$ is the second class Bessel function.

2) Case: $V=-V_{0}$

Now the wave function is

$$
\psi_{\nu}(\bar{\phi}, \beta)=C e^{i \nu \beta} K_{i \nu}\left(\lambda_{s} \sqrt{V_{0}} e^{-2 \bar{\phi}}\right),
$$

where $K_{i \nu}$ is the modified Bessel function. We can construct wormhole type solutions by means integrating over the separation constant $\nu$,

$$
\begin{aligned}
\psi_{W H}(\bar{\phi}, \beta) & =\int_{-\infty}^{+\infty} e^{i \nu(\beta+\mu)} K_{i \nu}\left(\lambda_{s} \sqrt{V_{0}} e^{\bar{\phi}}\right) d \nu \\
& =e^{-\lambda_{s} \sqrt{V_{0}} e^{\bar{\phi}} \cosh [2 \beta+\mu]} .
\end{aligned}
$$

where $\mu=$ const. For the noncommutative quantum cosmology model, we will assume the the "cartesian coordinates" $\bar{\phi}$ and $\beta$ of the Robertson-Walker minisuperspace obey a kind of commutation relation [ 9 ],

$$
[\bar{\phi}, \beta]=i \theta \text {. }
$$

This is a particular ansatz in these configuration coordinates. The deformation of minisuperspace can be studied in terms of Moyal [10] product,

$$
f(\bar{\phi}, \beta) \star g(\bar{\phi}, \beta)=f(\bar{\phi}, \beta) \exp \left[i \frac{\theta}{2}\left(\overleftarrow{\frac{\partial}{\partial \bar{\phi}}} \frac{\vec{\partial}}{\partial \beta}-\overleftarrow{\partial} \frac{\vec{\partial}}{\partial \beta} \frac{\vec{\phi}}{\partial \bar{\phi}}\right)\right] g(\bar{\phi}, \beta)
$$

Then the noncommutative WDW equation is

$$
\frac{1}{2 \lambda_{s}} \star\left[\frac{\partial^{2}}{\partial \bar{\phi}^{2}}-\frac{\partial^{2}}{\partial \beta^{2}}+\lambda_{s}^{2} V(\bar{\phi}, \beta) e^{-2 \bar{\phi}}\right] \star \psi(\phi, \beta)=0 .
$$

It is possible to reformulate this equation in terms of commutative variables and the ordinary product of functions, if new variables are introduced $\bar{\phi} \rightarrow \bar{\phi}-\frac{1}{2} \theta \Pi_{\beta}$ and $\beta \rightarrow \beta+\frac{1}{2} \theta \Pi_{\bar{\phi}}$. Therefore, the original equation changes, with a potential modified due to these new variables.

$$
V(\bar{\phi}, \beta) \star \psi(\bar{\phi}, \beta)=V\left(\bar{\phi}-\frac{1}{2} \theta \Pi_{\beta}, \beta+\frac{1}{2} \theta \Pi_{\bar{\phi}}\right) \psi(\bar{\phi}, \beta) .
$$


Then, we get

$$
\left[\frac{\partial^{2}}{\partial \bar{\phi}^{2}}-\frac{\partial^{2}}{\partial \beta^{2}}+\lambda_{s}^{2} V e^{-2 \bar{\phi}+\theta \Pi_{\beta}}\right] \psi(\bar{\phi}, \beta)=0 .
$$

In order to separate variables we propose the ansatz

$$
\psi(\bar{\phi}, \beta)=e^{\nu \beta} B(\bar{\phi}),
$$

the operator $\Pi_{\beta}$ in the exponential in equation (14) will shift the wave function by a factor

$$
\psi(\bar{\phi}, \beta-i \nu \theta)=e^{-2 i \nu \theta} \psi(\bar{\phi}, \beta)
$$

thus $B(\bar{\phi})$ must satisfy the equation

$$
\left[\frac{d^{2}}{d \bar{\phi}^{2}}+\nu^{2}+\lambda_{s}^{2} V e^{-2 \bar{\phi}-2 i \nu \theta}\right] F(\bar{\phi})=0
$$

1) $V=V_{0} e^{4 \bar{\phi}}$

The wave function is given by

$$
\psi(\bar{\phi}, \beta)=e^{i \nu \beta} Y_{i \nu}\left(\lambda_{s} \sqrt{V_{0}} e^{\bar{\phi}-i \nu \theta}\right),
$$

we can see that noncommutativity induces a difference of the arguments of the Bessel functions in the wave function.

2) $V=V_{0}$

Now the wave function is

$$
\psi(\bar{\phi}, \beta)=e^{i \nu \beta} K_{i \nu}\left(\lambda_{s} \sqrt{V_{0}} e^{-2 \bar{\phi}-i \nu \theta}\right)
$$

Now, in order to see a direct influence of parameter $\theta$ we construct the wave packet

$$
\psi(\bar{\phi}, \beta)=\int_{-\infty}^{\infty} e^{-a(\nu-b)^{2}} e^{i \nu \beta} K_{i \nu}\left(\lambda_{s} \sqrt{V_{0}} e^{-2 \bar{\phi}-i \nu \theta}\right) d \nu .
$$

In figures 1 and 2 we have plotted the square of the absolute value of the wave packets for different values of the noncommutativity parameter $\theta$ and the chosen values of $a=2$ and $b=3$. From the figure it is clear how important could be the existence of noncommutativity at the early stages od the Universe. At present times the experiment related to noncommutative quantum mechanics set a very small value for the parameter $\theta$, however those limits may not be applicable in quantum cosmology with the high energy regime.

\section{ACKNOWLEDGMENTS}

C Mora is supported by a COFAA-IPN grant.

[1] A. Connes, M. R. Douglas and A. Schwarz, JHEP 9802 (1998) 003, hep-th/9711162

[2] C. S. Chu and P. M. Ho NUcl. Phys. B550 (1999) 151; V. Schomerus, JHEP 98026 (1999) 030, hep-th/9903205 D. Bigatti and L. Susskind, hep-th/9908056 N. Seibert and E. Witten, JHEP 9809 (1999) 032, hep-th/9908142 .

[3] H. García-Compeán, O. Obregón and C. Ramírez, Phys. Rev. Lett. 57, 1015 (1998).

[4] Juan M. Romero and J. David Vergara, Mod.Phys.Lett. A18 (2003) 1673-1680, hep-th/0303064

[5] A. H. Chamseddine, J.Math.Phys. 44 (2003) 2534-2541, hep-th/0202137 V. O. Rivelles Noncommutative Field Theory and Gravity, hep-th/0212262

[6] J.M. Carmona, J.L. Cortes, J. Gamboa, F. Mendez, JHEP 0303 (2003) 058, hep-th/0301248

[7] L. O. Pimentel and C. Mora, work in preparation.

[8] M. Gasperini, J. Maharana and G. Veneziano, Nucl. Phys. B 472, 349 (1996).

[9] J. Gamboa, M. Loewe and J. C. Rojas, Phys. Rev. D62, 067901 (2001), hetp-th/0010220

[10] J.E. Moyal, Proc. Camb. Phil. Soc. 45 (1949) 99. 


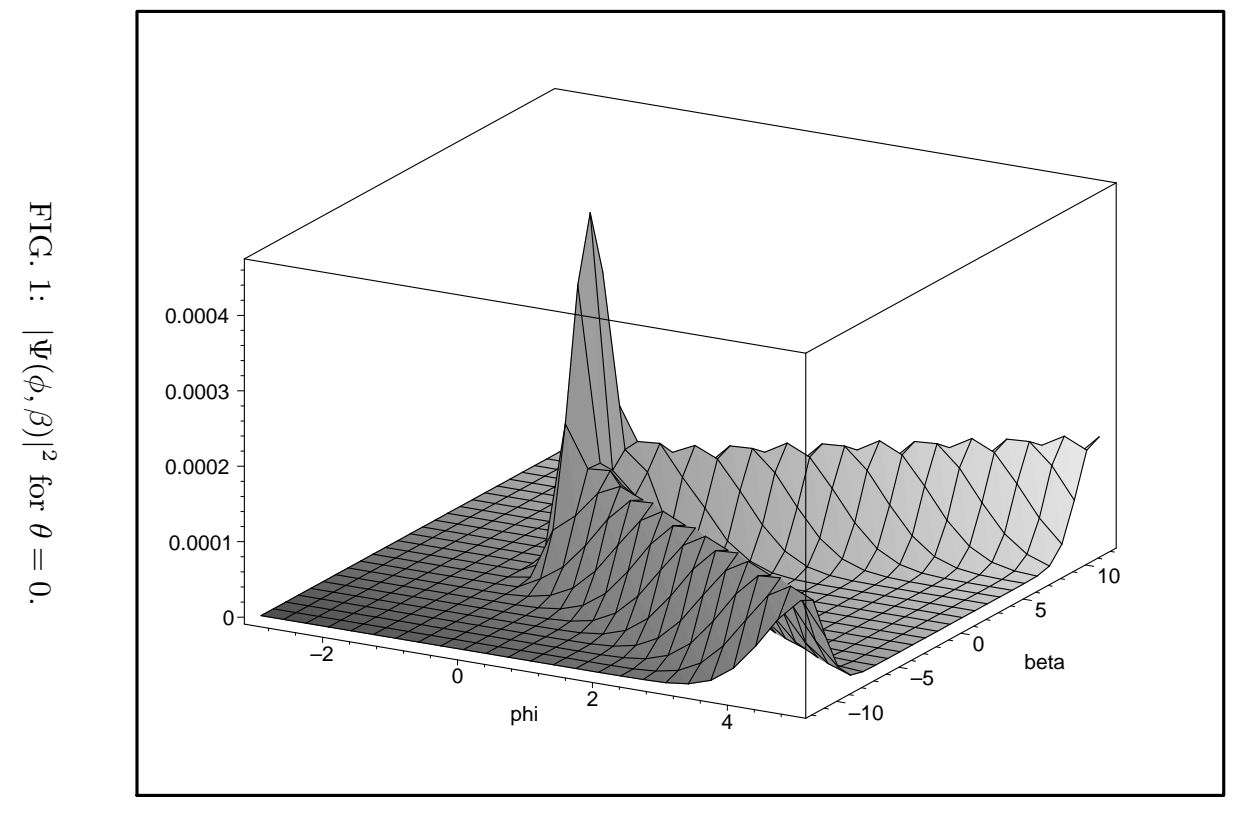




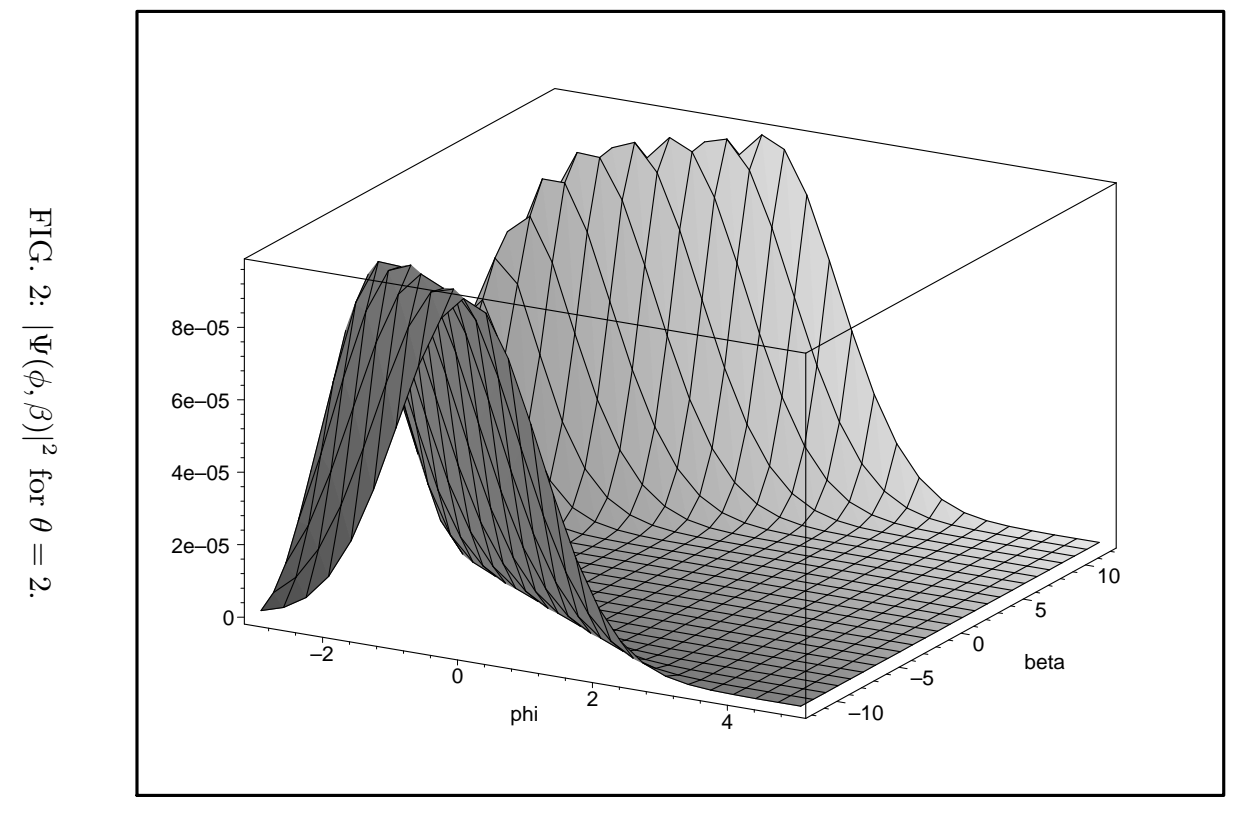

